Published in final edited form as:

Sleep Med. 2017 October ; 38: 85-91. doi:10.1016/j.sleep.2017.07.001.

\title{
Sleep and cognitive performance: Cross-sectional associations from the UK Biobank
}

\author{
Simon D. Kyle, PhD ${ }^{a}$, Claire E. Sexton, DPhil ${ }^{b}$, Bernd Feige, $\mathrm{PhD}^{\mathrm{c}}$, Annemarie Luik, $\mathrm{PhD}^{\mathrm{a}}$, \\ Jacqueline Lane, $\mathbf{P h D}^{\mathrm{d}, \mathrm{e}, \mathrm{f}}$, Richa Saxena, $\mathbf{P h D}^{\mathrm{d}, \mathrm{e}, \mathrm{f}, \mathrm{g}}$, Simon G. Anderson, $\mathbf{P h D}^{\mathrm{h}}$, David A. \\ Bechtold, PhD', William Dixon, PhDj, Max Little, PhD $^{\mathrm{k}, l}$, David Ray, PhD ${ }^{\mathrm{m}}$, Dieter Riemann, \\ $\mathbf{P h D}^{c}$, Colin A. Espie, PhDa, Martin K. Rutter, $\mathbf{P h D}^{\mathrm{m}, \mathrm{n}}$, and Kai Spiegelhalder, $\mathbf{P h D}^{\mathrm{c}}$ \\ aSleep and Circadian Neuroscience Institute (SCNi), Nuffield Department of Clinical \\ Neurosciences, University of Oxford, UK \\ bFMRIB Centre, Nuffield Department of Clinical Neurosciences, John Radcliffe Hospital, \\ University of Oxford, UK \\ ${ }^{\circ}$ Clinic for Psychiatry and Psychotherapy, Medical Centre - University of Freiburg, Faculty of \\ Medicine, University of Freiburg, Germany \\ ${ }^{d}$ Center for Human Genetic Research Massachusetts General Hospital, Boston, Massachusetts, \\ USA
}

eAnesthesia, Critical Care and Pain Medicine, Massachusetts General Hospital and Harvard Medical School, Boston, Massachusetts, USA

fProgram in Medical and Population Genetics, Broad Institute, Cambridge, Massachusetts, USA gDepartment of Anesthesia, Division of Sleep and Circadian Disorders, Brigham and Women's Hospital, Boston, Massachusetts, USA

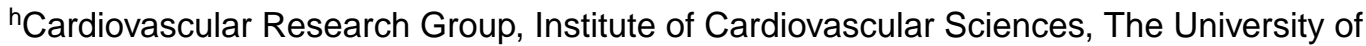
Manchester, Manchester, UK

'Faculty of Life Sciences, University of Manchester, Manchester, UK

jCentre for Musculoskeletal Research Institute of Inflammation and Repair, The University of Manchester, Manchester, UK

kEngineering and Applied Science, Aston University, Birmingham, UK

'Media Lab, Massachusetts Institute of Technology, Cambridge, MA, USA

${ }^{m}$ Centre for Endocrinology and Diabetes, Institute of Human Development, University of Manchester, UK

Correspondence to: Simon D. Kyle, PhD, Sleep and Circadian Neuroscience Institute (SCNi), Nuffield Department of Clinical Neurosciences, Sir William Dunn School of Pathology, University of Oxford, South Parks Road, Oxford, OX1 3RE, Tel. 01865 618675. simon.kyle@ndcn.ox.ac.uk.

Disclosure: AIL receives a salary from Big Health Ltd. CAE is shareholder and co-founder of Big Health Ltd. MKR has received research support from GSK and Novo Nordisk, acted as consultant for GSK, Roche, and Cell Catapult, and is stockholder in GSK. RS has received salary from and is stockholder in Astrazeneca and Surface Oncology. SDK has previously acted as consultant for Big Health Ltd. All remaining authors declare no potential conflicts of interest. 
nManchester Diabetes Centre, Central Manchester University Hospitals NHS Foundation Trust, Manchester Academic Health Science Centre, Manchester, UK

\section{Abstract}

Objective-The relationship between insomnia symptoms and cognitive performance is unclear, particularly at the population level. We conducted the largest examination of this association to date through analysis of the UK Biobank, a large population-based sample of adults aged 40-69 yrs. We also sought to determine associations between cognitive performance and self-reported chronotype, sleep medication use, and sleep duration.

Methods-This cross-sectional, population-based study involved 477,529 participants, comprising 133,314 with frequent insomnia symptoms (age: $57.4 \pm 7.7 \mathrm{yrs} ; 62.1 \%$ female) and 344,215 controls without (age: $56.1 \pm 8.2$ yrs; $52.0 \%$ female). Cognitive performance was assessed through a touchscreen test battery probing reasoning, basic reaction time, numeric memory, visual memory and prospective memory. Adjusted models included relevant demographic, clinical and sleep variables.

Results-Frequent insomnia symptoms were associated with cognitive impairment in unadjusted models, however these effects were reversed after full adjustment, leaving those with frequent insomnia symptoms showing statistically better cognitive performance over those without. Relative to intermediate chronotype, evening chronotype was associated with superior task performance, while morning chronotype was associated with the poorest performance. Sleep medication use and both long ( $>9 \mathrm{hrs})$ and short ( $<7 \mathrm{hrs})$ sleep duration were associated with impaired performance.

Conclusions-Our results suggest that after adjustment for potential confounding variables, frequent insomnia symptoms may be associated with a small statistical advantage, which is unlikely to be clinically meaningful, on simple neurocognitive tasks. Further work is required to examine mechanistic underpinnings of an apparent evening chronotype advantage in cognitive performance, as well as impairment associated with morning chronotype, sleep medication use, and sleep duration extremes.

\section{Keywords}

insomnia; cognitive performance; chronotype; sleep duration; sleep medication

\section{Introduction}

Insomnia disorder is defined as persistent difficulties with sleep initiation and/or maintenance, resulting in significant impairment to daytime functioning. At the symptom level, insomnia affects up to one-third of the adult population, while persistent insomnia disorder affects approximately $10-12 \%$ and is associated with increased risk for cardiovascular disease, depression and early mortality. ${ }^{1,2}$ Both daytime functioning and quality of life are known to be severely affected in those with insomnia and often drive treatment-seeking. ${ }^{3-5}$ More specifically, previous work shows that the most commonly cited areas of daytime dysfunction are problems with fatigue, work performance, cognitive 
performance and emotion regulation ${ }^{6}$. Insomnia has also been associated with a range of serious and non-serious sleep-related accidents ${ }^{7}$.

While experimental sleep loss engenders reliable cognitive impairment, particularly for vigilance, complex attention and working memory, ${ }^{8}$ there has been comparatively little work on insomnia. In general, the field has been characterised by mixed findings, with some studies showing impairment and others failing to observe differences from controls. ${ }^{9}$ Nevertheless, meta-analytic data suggest that patients exhibit reliable impairments in tasks probing episodic memory, working memory and problem solving, with small-to-medium effect sizes. ${ }^{10}$ Recent, well-controlled studies find evidence of insomnia-related impairments in switching of attention and working memory, ${ }^{11}$ and sustained attention and episodic memory. ${ }^{12}$ However, there continues to be conflicting findings in the insomnia literature ${ }^{13-15}$ and studies generally recruit small samples of patients with "primary insomnia", who are otherwise healthy.

Larger epidemiology-based studies of insomnia symptoms and cognitive performance similarly display mixed results: showing evidence of impairment, ${ }^{16}$ no evidence of impairment, ${ }^{17}$ or impairment only for specific insomnia sub-groups. ${ }^{15,18}$ To our knowledge, no study has investigated insomnia symptoms and cognitive performance in a large population-based sample of middle-aged adults, with a standardised test battery, while simultaneously appraising the influence of other important sleep variables, including chronotype, sleep duration, and sleep medication.

The aim of the present study was to conduct the largest investigation of insomnia symptoms and cognitive performance to date, through analysis of UK biobank data. The UK biobank is a large population-based study of $>500,000$ adults aged between 40 and 69 , providing a unique opportunity to assess associations in groups of poor and good sleepers, and to adequately control for the influence of several potential confounding variables. We hypothesised that insomnia would be independently associated with impairments in all measures of cognition (reasoning, basic reaction time, numeric memory, visual memory and prospective memory) after controlling for potential confounding variables. As a secondary aim we examined associations between cognitive performance and chronotype, sleep medication use, and self-reported sleep duration.

\section{Methods}

\section{Participants}

Details of the UK Biobank are available elsewhere. ${ }^{19}$ In brief, adults aged 40 to 69 years who were registered with the UK National Health Service and living within 25 miles of a study assessment centre were invited to participate. Approximately 9 million invitations led to a final sample of 501,718 participants. For the purposes of the present study, participants were excluded if they self-reported a neurological condition (e.g., neurodegenerative disease, stroke, head injury, epilepsy; $\mathrm{n}=22,065)$, had a diagnosis of sleep-disordered breathing $(\mathrm{n}=1,511)$, or had incomplete data for insomnia symptoms ( $\mathrm{n}=613)$, leaving a total of 477,529 . Twenty-eight percent of the sample reported frequent insomnia symptoms $(\mathrm{n}=133,314$; mean age $=57.4 \mathrm{yrs}, \mathrm{SD}=7.7 \mathrm{yrs} ; 62.1 \%$ female $)$, while the remaining $72 \%$ of 
participants made up the comparison group $(\mathrm{n}=344,215$; mean age $=56.1 \mathrm{yrs}, \mathrm{SD}=8.2 \mathrm{yrs}$; $52.0 \%$ female). This comparison group was comprised of those reporting insomnia symptoms "sometimes" [48\%] and "never/rarely" [28\%].

\section{Procedure and measurements}

All UK Biobank research procedures were approved by the NHS National Research Ethics Service (Ref. 11/NW/0382). All participants gave written informed consent. Assessments took place at 22 centres across England, Scotland and Wales between 2006 and 2010. Questionnaires and cognitive assessments were administered in a standardised order via a computerized touchscreen interface, followed by a face-to-face interview with a research nurse to obtain additional data. Assessments of sleep-related variables and cognitive performance took place in a single visit lasting approximately 90 minutes.

\section{Sleep-related variables}

To assess insomnia symptoms, participants were asked "Do you have trouble falling asleep at night or do you wake up in the middle of the night?" with responses "never/rarely", "sometimes", and "usually". Participants were categorised as having frequent insomnia symptoms if they answered "usually" to this question, while the remaining participants made up the control group without frequent insomnia symptoms. Chronotype was assessed using the following question, "Do you consider yourself to be"...: "definitely a "morning' person", "more a 'morning' than 'evening' person", "more an 'evening' than 'morning' person", "definitely an 'evening' person". For the purposes of the present study, we collapsed the two middle responses into an "intermediate" chronotype category, permitting comparisons with the 'definitely morning' and 'definitely evening' groups. Sleep duration was recorded as number of reported hours to the following question "About how many hours sleep do you get in every 24 hours? (please include naps)". Given previously established U-shape relationships with health and cognition, ${ }^{20}$ we categorised sleep duration into short $(<7 \mathrm{hrs})$, normal (7-9 hrs) and long (>9hrs) based on recent guidelines. ${ }^{21}$ Finally, as a manipulation check to support group categorisation (insomnia symptoms vs. no insomnia symptoms), subjective daytime impairment was coded based on answers to the following question: "Over the past two weeks, how often have you felt tired or had little energy?", with the following response options: "not at all", "several days", "more than half the days", or "nearly every day".

\section{Cognitive Performance}

Five cognitive measures were administered via computerised touchscreen interface. ${ }^{22}$ Time to complete all five cognitive tests was approximately 15 minutes. The tests were designed specifically for UK Biobank, in order to allow administration at scale without examiner supervision. The tasks show evidence of an underlying performance factor and good stability over time, with the exception of visual memory performance which has a comparatively lower intraclass correlation coefficient. ${ }^{22}$

Briefly, the assessments included: 
- $\quad$ reasoning: this task assessed the ability to solve thirteen verbal and numeric reasoning problems. Each problem had five possible response options. The dependent variable was total number of correct answers given (range 0-13) within a two-minute period, with higher scores indicating better performance;

- basic reaction time: this task was delivered in the style of the card game, 'snap', and requested participants to respond with a button press when they detected the appearance of a matching pair of symbols. The dependent variable was mean response time in milliseconds across 12 matching-pair trials. RT values were logtransformed due to skewed distribution $(\ln \mathrm{x})$;

- $\quad$ numeric short-term memory: In this task a string of numbers were presented on the screen which subsequently disappeared. Participants were asked to then enter the number string from memory, in reverse order, via keypad response. The dependent variable was maximum string length recalled correctly (range: 0-12), with higher scores indexing better performance. The test was discontinued after five successive incorrect responses at string length $=2$;

- $\quad$ visual memory: In this task six card-pairs of symbols were presented on-screen in a random pattern. Cards were then turned face down on the screen and participants were asked to locate as many symbol pairs as possible in as few attempts as possible. The dependent variable was the number of errors made during pairs matching (range 0-146), which was log-transformed due to skewed distribution and zero-inflation $(\ln (\mathrm{x}+1))$;

- $\quad$ prospective memory: In this task participants were asked to remember to carry out a pre-planned instruction after a filled interval. At the beginning of the test battery they were presented with the following instruction: "At the end of the games we will show you four coloured symbols and ask you to touch the blue square. However, to test your memory, we want you to actually touch the orange circle instead." If participants remembered to touch the orange circle on first attempt they were coded as "correct" (1), while those failing to do so were set to 0 .

\section{Demographic data}

Demographic data included age, sex and neighborhood-level socioeconomic status as measured by the Townsend index of material deprivation. For statistical analyses, socioeconomic status was log-transformed due to skewed distribution using an ' $\ln (\mathrm{x}+7)$ ' equation (minimum of non-transformed index: -6.26). Educational qualifications were recorded, and were dichotomised according to whether or not participants held a college / university degree. We also included body mass index (BMI) as a covariate in adjusted analyses.

\section{Medication and clinical data}

Current medications were self-reported to the research nurse, and participants were dichotomised according to whether or not they were taking sleep medication (sedatives and hypnotics), any other psychotropic medication (mood stabilisers, antidepressants, 
antipsychotics), or antihypertensive medication (ACE inhibitors, angiotensin II antagonists, beta blockers, calcium channel blockers, diuretics). Current depressive symptoms were assessed using the following question, "Over the past two weeks, how often have you felt down, depressed or hopeless?" with the following response options: "not at all", "several days", "more than half the days" or "nearly every day". For the purpose of the present analyses, those scoring "several days", "more than half the days", or "nearly every day" were coded in the "depressive symptoms" category, while those scoring "not at all" were considered in the "no depressive symptoms" category. In addition, participants were dichotomised according to whether or not they reported hypertension or any cardiovascular disease.

\section{Analyses}

Descriptive data presentation included mean values and standard deviations, as well as the proportion of the sample reporting specific questionnaire response options. Questionnaire response options, 'do not know' or 'prefer not to answer', were handled as missing values. For cognitive dependent variables, sample size varied across tests because the reasoning and prospective memory tests were added after commencement of data collection, while the numeric memory task was included from the outset but subsequently removed due to time constraints.

In a first step (unadjusted analyses, model 1), the association between insomnia status (frequent insomnia symptoms vs. those without frequent insomnia symptoms) and cognitive performance was analysed using four linear models, with insomnia status as the single predictor variable and reasoning, basic reaction time, numeric memory and visual memory as dependent variables. Association between insomnia status and prospective memory performance was investigated using a logistic model. In a second step (model 2), age, sex, socioeconomic status and education were added as covariates in the linear and logistic models described in model 1. In a third step (model 3), chronotype (with the intermediate type as the reference category), sleep medication use, BMI as a continuous variable, hypertension, antihypertensive medication, cardiovascular disease, depressive symptoms, and psychotropic medication were added as further covariates. In a final step (model 4), sleep duration (with 7-9 hrs as the reference category) was inserted as an additional predictor of cognitive performance. We therefore report unadjusted and multivariateadjusted relationships between insomnia status and cognitive outcomes. For chronotype, sleep duration and sleep medication we report only the fully adjusted relationships (model 4) controlling for all demographic, clinical and remaining sleep variables. Given that we analysed five cognitive tests, the alpha level was set at $p<0.01$ for all analyses.

\section{Results}

\section{Sample description}

Sociodemographic data for the sample are presented in Table 1 . Those with frequent insomnia symptoms were older and more likely to be female, reported shorter sleep duration, were more likely to report using sleep medication, were less likely to hold a university or college degree, were from a lower socioeconomic background, had a higher 
BMI, were more likely to report hypertension, use of antihypertensive medication or cardiovascular disease, were more likely to report recent depressive symptoms and were more likely to report taking psychotropic medication (all $\mathrm{p}<0.001$ ).

\section{Association between insomnia symptoms and cognitive performance}

Results from statistical models are presented in Table 2. In unadjusted analyses (model 1), frequent insomnia symptoms were associated with worse performance on the reasoning test, basic reaction time test, numeric memory test, and visual memory test. There was no statistically significant association between insomnia status and performance on the prospective memory test after correction for multiple testing.

In age, sex, socioeconomic status and education-adjusted analyses (model 2), insomnia was no longer associated with performance on the reasoning test or numeric memory test. Adjustment revealed that insomnia was associated with statistically better performance on the basic reaction time test, visual memory test and prospective memory test.

Further adjustment for clinical and sleep-related variables (models 3 and 4) showed that frequent insomnia symptoms were associated with better performance on the reasoning test, basic reaction time test, visual memory test, and prospective memory test, compared to those without frequent insomnia symptoms. There was no statistically significant independent association between insomnia status and performance on the numeric memory test.

\section{Chronotype, sleep medication, and sleep duration}

$27.0 \%$ of the sample were categorized as morning chronotype, $64.0 \%$ as intermediate, and $8.9 \%$ as evening chronotype. In fully adjusted analyses (model 4; see Table 3), evening chronotype was associated with superior task performance on the reasoning test, basic reaction time test, visual memory test, and prospective memory test, relative to those with an intermediate chronotype, while there was no significant association with task performance on the numeric memory test. On the contrary, morning chronotype, relative to intermediate chronotype, was associated with poorer performance on the reasoning test, basic reaction time test, numeric memory test, visual memory test, and prospective memory test.

Sleep medication use was associated with reduced performance on the reasoning test, basic reaction time test, and prospective memory test, but not on the numeric memory test or visual memory test.

$73.7 \%$ of the sample reported sleep duration between 7 and 9 hours, $24.6 \%$ reported sleep duration $<7$ hours, while $1.7 \%$ reported sleep duration $>9$ hours. Both short ( $<7 \mathrm{hrs})$ and long ( $>9 \mathrm{hrs}$ ) sleep duration were associated with poorer performance on the reasoning test, basic reaction time test, numeric memory test, visual memory test, and prospective memory test.

\section{Discussion}

The principal aim of the present study was to examine cross-sectional associations between insomnia symptoms and cognitive performance in a large population-based sample. We also 
sought to assess relationships between cognitive performance and sleep duration, chronotype and sleep medication use. Prevalence, demographic and comorbidity profile of those with insomnia symptoms was consistent with previous epidemiological investigations. ${ }^{1}$ Unadjusted analyses revealed that those with frequent insomnia symptoms displayed impairment across all cognitive tasks (except prospective memory); however, intermediate adjustment for demographic variables rendered these associations non-significant or reversed their direction. Full adjustment (model 4) for demographic, medical and sleeprelated variables left those with frequent insomnia symptoms with a small, but statistically significant advantage over those without insomnia for all cognitive measures (except numeric memory). On the other hand, sleep medication use, as well as both long ( $>9 \mathrm{hrs)}$ and short $(<7 \mathrm{hrs})$ sleep duration were independently associated with cognitive impairment, while evening chronotype was linked to better task performance.

Our study represents the largest investigation of insomnia symptoms and cognitive performance to date and is consistent with results from population-based studies which similarly failed to observe negative relationships, ${ }^{15,17}$ whilst conflicting with others. ${ }^{16,24}$ Clearly our study stands apart with respect to sample size and ability to control for the influence of multiple confounding sleep and non-sleep variables. The apparent small, but statistical advantage of those with insomnia versus those without was unexpected and is unlikely to be clinically meaningful [note, effect size differences in SD units $=.044$ (reasoning), .035 (reaction time) and .022 (visual memory)]. Nevertheless, it is worth reflecting on what factors may contribute to these consistent effects. Typical of large epidemiological-based studies, there remains the possibility of unmeasured confounding that may explain group differences. We note that a recent report on UK Biobank data similarly found, unexpectedly, that those with a history of probable single episode depression or probable recurrent depression displayed a small but statistically significant cognitive advantage over controls. ${ }^{23}$ It is possible that our samples are overlapping.

There may also be trait factors relevant to poor sleepers that aid compensation and/or performance facilitation. For example, hyperarousal across cognitive, emotional and neurophysiological levels is characteristic of insomnia ${ }^{25}$ and has been suggested to support cognitive performance on relatively brief tasks with low cognitive load. ${ }^{26}$ In support of this, at least two studies have observed quicker reaction times on basic vigilance tasks in insomnia patients relative to controls. ${ }^{27,28}$ Hyperarousal has in turn been proposed to hinder performance on more cognitively challenging tasks ${ }^{29,30}$ and it may be that tests used in the present study were not of sufficient sensitivity and/or complexity to unmask insomniarelated impairment. ${ }^{31}$

Another possibility is that unmeasured personality traits known to be associated with insomnia may contribute to enhanced cognitive performance. We and others have shown that those with insomnia often display high levels of perfectionism, captured through questionnaire and behavioural measures. ${ }^{32-36}$ Preliminary work suggests that high levels of perfectionism may confer advantage on specific task-dependent variables under certain conditions. ${ }^{37}$ However, studies have also revealed that perfectionistic traits (and associated processes, like rumination) may be obstructive to task performance. ${ }^{38}$ Thus, the drivers behind the observed statistical advantage remain speculative and unclear, but could involve 
both etiological and methodological factors. Of note, supplementary analysis (see supplementary Table 1) comparing those reporting insomnia symptoms "sometimes" with those reporting insomnia symptoms "never/rarely" did not reveal group differences in adjusted models. However, consistent with results presented above, those with frequent insomnia symptoms performed better relative to those reporting insomnia symptoms "never/ rarely". There is, therefore, no clear dose-response effect between frequency of insomnia symptoms and cognitive performance.

\section{Chronotype, sleep medication, and sleep duration}

While sleep disturbance was not independently associated with task impairment we observed associations between cognitive performance and chronotype, sleep medication use and sleep duration. Evening chronotype was associated with better performance across all tasks (except numeric memory), while those endorsing a morning preference performed worse than the intermediate group. It must be noted that effect size differences for evening and morning chronotypes, relative to the intermediate group, were in the very small range (SD units difference: .023-.116). This is the first time that such relationships have been observed in a large population-based sample with a standardized test battery, supporting a range of smaller studies reporting on academic achievement, salary and intelligence outcomes. ${ }^{39-42}$ We note, however, that our findings are at odds with evidence from diffusion tensor imaging showing white matter deficits in frontal and temporal lobes, cingulate gyrus and corpus callosum in evening types. ${ }^{43}$ Although we were unable to investigate time of testing in relation to chronotype and performance, assessments took place during standard office hours and therefore would be expected to work against evening types, rather than account for superior performance. Findings are consistent with our recent biobank-GWAS which found that genetic loci underlying eveningness were associated with higher educational attainment. ${ }^{44}$ Importantly, we find chronotype-performance associations independent of educational background and socioeconomic status. Theoretical conceptualisations of an evening chronotype advantage and morning type impairment remain under-developed ${ }^{45}$ but our work suggests these are likely to be reliable associations at the population level.

We observed that sleep medication and long/short sleep duration are independently associated with poorer cognitive performance (SD units difference: .048-.132 [sleep medication]; .013-.069 [short sleep duration]; .094-.269 [long sleep duration]). These effects have been shown across a range of study designs and samples ${ }^{17,20,46,47}$ but our study is particularly noteworthy given our ability to partial out the effect of potential confounders, including both sleep and non-sleep variables. We were not able to record history or duration of sleep medication use but both acute and chronic use of benzodiazepines/benzodiazepine receptor agonists is known to be associated with cognitive and psychomotor impairment, ${ }^{47}$ while their discontinuation in chronic users is associated with improved cognitive status. ${ }^{48}$ Sleep medication use appears to be more robustly associated with cognitive impairment than insomnia symptoms, which may have important clinical implications. It of course remains possible that extremes of sleep duration and sleep medication act as proxies for underlying poor health, accounting for associated impairment. Future, longitudinal efforts are needed to examine this possibility more directly, incorporating a greater number of potential health- 
related confounds. Finally, examination of associations between sleep traits and functional and structural neuroimaging parameters is now required to shed light on putative brain mechanisms underlying performance profiles.

\section{Limitations}

Our study has several important caveats. First, this was a cross-sectional study and therefore our findings cannot elucidate causality. Second, our cognitive dependent variables were created for brief completion on large numbers of participants and hence have not undergone formal validation. In partial mitigation, principal components analyses support an expected underlying performance (g) factor across tasks, and test-retest suggests good stability for most task dependent variables. ${ }^{22}$ Moreover, associations with variables in the expected direction (e.g. with age, education), evidence of impairment in those with psychiatric disorder, ${ }^{23}$ and associations with several genetic variants ${ }^{49}$ provide further support of task validity. However, tasks were brief and did not parametrically manipulate cognitive load, and the battery did not sample additional domains of cognition; potentially contributing to limited sensitivity for insomnia-related impairment. Third, our sleep trait predictor variables were limited by their low-resolution, reflecting just single item self-report questions. For example, our sleep duration estimate included daytime naps and therefore we could not tease apart the contribution of night and day-time sleep, which may have influenced group categorization and, by extension, associations with our dependent variables of interest. We could not investigate subtypes of insomnia that may have unique relationships with cognitive impairment. ${ }^{16}$ Specifically, we were not able to categorise participants into insomnia disorder since we lacked information on chronicity of sleep problems, quantitative criteria (e.g. minutes for sleep-onset latency, wake-time during the night), attribution for daytime cognitive impairment, or help-seeking. Greater precision around these factors and objective sleep data may have identified sub-groups of participants with cognitive impairment. $12,15,18,30,50$ Our results therefore can only speak to frequent insomnia symptoms at the population level. Fourth, our sample age was restricted to those predominantly in middleage. It may be that insomnia confers greatest risk to cognitive impairment in elderly samples. ${ }^{24}$ Fifth, while the UK biobank represents a large and unique resource, method of recruitment and low response rate (5.5\%) may have resulted in selection biases, potentially limiting generalizability to the broader UK population. ${ }^{51}$ Sixth, sample size differed across statistical models owing to missing data for specific covariates of interest. However, we note that when analyses are restricted to complete data across all models, findings remain unchanged (see Supplementary Table 2). Finally, our analyses are based exclusively on Gaussian linear models, and we have not tested whether nonlinear and/or non-Gaussian models would reveal more nuanced associations between our predictor and outcome variables.

\section{Conclusion}

In our large UK sample frequent insomnia symptoms were not independently associated with cognitive impairment. Indeed, contrary to our hypotheses, we found a small statistical advantage for those with frequent insomnia symptoms over those without, which is unlikely to be clinically meaningful. Our data in no way undermine subjective daytime reports of those with poor sleep, since task performance may not fully map onto daytime 
phenomenology. ${ }^{4,10}$ We cannot exclude the possibility that those with insomnia disorder or discrete sub-groups of insomnia do not exhibit reliable performance impairment. Reliability of, and mechanisms underpinning, the small evening chronotype advantage and morning chronotype disadvantage require further examination, including consideration of time of testing. Finally, our results corroborate cognitive risk associated with sleep medication use as well as both long and short sleep duration.

\section{Supplementary Material}

Refer to Web version on PubMed Central for supplementary material.

\section{Acknowledgments}

This research has been conducted using the UK Biobank Resource.

\section{References}

1. Morin CM, Benca R. Chronic insomnia. Lancet. 2012; 379:1129-41. [PubMed: 22265700]

2. Morin CM, Drake CL, Harvey AG, et al. Insomnia disorder. Nat Rev Dis Primers. 2015; 1:15026. [PubMed: 27189779]

3. Kyle SD, Morgan K, Espie CA. Insomnia and health-related quality of life. Sleep Med Rev. 2010; 14:69-82. [PubMed: 19962922]

4. Kyle SD, Espie CA, Morgan K. “... Not just a minor thing, it is something major, which stops you from functioning daily": quality of life and daytime functioning in insomnia. Behav Sleep Med. 2010; 8:123-40. [PubMed: 20582756]

5. Espie CA, Kyle SD, Hames P, Cyhlarova E, Benzeval M. The daytime impact of DSM-5 insomnia disorder: comparative analysis of insomnia subtypes from the Great British Sleep Survey. J Clin Psychiatry. 2012; 73:e1478-84. [PubMed: 23290331]

6. Kyle SD, Crawford MR, Morgan K, Spiegelhalder K, Clark AA, Espie CA. The Glasgow Sleep Impact Index (GSII): a novel patient-centred measure for assessing sleep-related quality of life impairment in Insomnia Disorder. Sleep Med. 2013; 14:493-501. [PubMed: 23347908]

7. Léger D, Bayon V, Ohayon MM, et al. Insomnia and accidents: cross-sectional study (EQUINOX) on sleep-related home, work and car accidents in 5293 subjects with insomnia from 10 countries. J Sleep Res. 2014; 23:143-52. [PubMed: 24237855]

8. Lim J, Dinges DF. A meta-analysis of the impact of short-term sleep deprivation on cognitive variables. Psychol Bull. 2010; 136:375-89. [PubMed: 20438143]

9. Shekleton JA, Rogers NL, Rajaratnam SM. Searching for the daytime impairments of primary insomnia. Sleep Med Rev. 2010; 14:47-60. [PubMed: 19963414]

10. Fortier-Brochu E, Beaulieu-Bonneau S, Ivers H, Morin CM. Insomnia and daytime cognitive performance: a meta-analysis. Sleep Med Rev. 2012; 16:83-94. [PubMed: 21636297]

11. Shekleton JA, Flynn-Evans EE, Miller B, et al. Neurobehavioral performance impairment in insomnia: relationships with self-reported sleep and daytime functioning. Sleep. 2014; 37:107-16. [PubMed: 24470700]

12. Fortier-Brochu E, Morin CM. Cognitive impairment in individuals with insomnia: clinical significance and correlates. Sleep. 2014; 37:1787-98. [PubMed: 25364074]

13. Lovato N, Lack L, Wright H, Cant M, Humphreys J. Working memory performance of older adults with insomnia. J Sleep Res. 2013; 22:251-7. [PubMed: 23171327]

14. Sivertsen B, Hysing M, Wehling E, et al. Neuropsychological performance in older insomniacs. Neuropsychol Dev Cogn B Aging Neuropsychol Cogn. 2013; 20:34-48. [PubMed: 22490041]

15. Goldman-Mellor S, Caspi A, Gregory AM, Harrington H, Poulton R, Moffitt TE. Is insomnia associated with deficits in neuropsychological functioning? Evidence from a population-based study. Sleep. 2015; 38:623-31. [PubMed: 25348123] 
16. Ling A, Lim ML, Gwee X, Ho RC, Collinson SL, Ng TP. Insomnia and daytime neuropsychological test performance in older adults. Sleep Med. 2016; 17:7-12. [PubMed: 26847967]

17. Kronholm E, Sallinen M, Suutama T, Sulkava R, Era P, Partonen T. Self-reported sleep duration and cognitive functioning in the general population. J Sleep Res. 2009; 18:436-46. [PubMed: 19732318]

18. Fernandez-Mendoza J, Calhoun S, Bixler EO, et al. Insomnia with objective short sleep duration is associated with deficits in neuropsychological performance: a general population study. Sleep. 2010; 33:459-65. [PubMed: 20394314]

19. Allen NE, Sudlow C, Peakman T, Collins R. UK biobank data: come and get it. Sci Transl Med. 2014; 6:224ed4.

20. Lo JC, Groeger JA, Cheng GH, Dijk D-J, Chee MWL. Self-reported sleep duration and cognitive performance in older adults: a systematic review and meta-analysis. Sleep Med. 2016; 17:87-98. [PubMed: 26847980]

21. Watson NF, Badr MS, Belenky G, et al. Recommended amount of sleep for a healthy adult: a joint consensus statement of the American Academy of Sleep Medicine and Sleep Research Society. Sleep. 2015; 38:843-4. [PubMed: 26039963]

22. Lyall DM, Cullen B, Allerhand M, et al. Cognitive Test Scores in UK Biobank: Data Reduction in 480,416 Participants and Longitudinal Stability in 20,346 Participants. PLoS One. 2016; 11:e0154222. [PubMed: 27110937]

23. Cullen B, Nicholl BI, Mackay DF, et al. Cognitive function and lifetime features of depression and bipolar disorder in a large population sample: Cross-sectional study of 143,828 UK Biobank participants. Eur Psychiatry. 2015; 30:950-8. [PubMed: 26647871]

24. Nebes RD, Buysse DJ, Halligan EM, Houck PR, Monk TH. Self-reported sleep quality predicts poor cognitive performance in healthy older adults. J Gerontol B Psychol Sci Soc Sci. 2009; 64:180-7. [PubMed: 19204069]

25. Riemann D, Spiegelhalder K, Feige B, et al. The hyperarousal model of insomnia: a review of the concept and its evidence. Sleep Med Rev. 2010; 14:19-31. [PubMed: 19481481]

26. Horne J. Primary insomnia: a disorder of sleep, or primarily one of wakefulness? Sleep Med Rev. 2010; 14:3-7. [PubMed: 19962332]

27. Altena E, Van Der Werf YD, Strijers RL, Van Someren EJ. Sleep loss affects vigilance: effects of chronic insomnia and sleep therapy. J Sleep Res. 2008; 17:335-43. [PubMed: 18844819]

28. Edinger JD, Fins AI, Sullivan RJ Jr, Marsh GR, et al. Do our methods lead to insomniacs' madness?L Daytime testing after laboratory and home-based polysomnographic studies. Sleep. 1997; 20:1127-34. [PubMed: 9493922]

29. Cellini N, de Zambotti M, Covassin N, Sarlo M, Stegagno L. Working memory impairment and cardiovascular hyperarousal in young primary insomniacs. Psychophysiology. 2014; 51:206-14. [PubMed: 24571027]

30. Edinger JD, Means MK, Krystal AD. Does physiological hyperarousal enhance error rates among insomnia sufferers? Sleep. 2013; 36:1179-86. [PubMed: 23904678]

31. Espie CA, Kyle SD. Towards an improved neuropsychology of poor sleep? Sleep. 2008; 31:591-2. [PubMed: 18517027]

32. Fabbri M, Tonetti L, Martoni M, Natale V. Remember to do: insomnia versus control groups in a prospective memory task. Behav Sleep Med. 2015; 13:231-40. [PubMed: 24611555]

33. Regen W, Hertenstein E, Weil P, et al. Perfectionistic tendencies in insomnia patients' behavior during psychometric testing. Behav Sleep Med. 2015; 13:387-94. [PubMed: 24925081]

34. Spiegelhalder K, Regen W, Kyle SD, et al. Time will tell: a retrospective study investigating the relationship between insomnia and objectively defined punctuality. J Sleep Res. 2012; 21:264-9. [PubMed: 21981420]

35. Azevedo MH, Bos SC, Soares MJ, et al. Longitudinal study on perfectionism and sleep disturbance. World J Biol Psychiatry. 2010 Mar; 11(2 Pt 2):476-85. [PubMed: 20218803]

36. Jansson-Fröjmark M, Linton SJ. Is perfectionism related to pre-existing and future insomnia? A prospective study. Br J Clin Psychol. 2007; 46:119-24. [PubMed: 17472206] 
37. Desnoyers A, Arpin-Cribbie C. Examining cognitive performance: Do perfectionism and rumination matter? Pers Individ Differ. 2015; 76:94-8.

38. Stoeber J, Eysenck MW. Perfectionism and efficiency: Accuracy, response bias, and invested time in proof-reading performance. J Res Pers. 2008; 42:1673-8.

39. Piffer D, Ponzi D, Sapienza P, Zingales L, Maestripier D. Morningness-eveningness and intelligence amongh high-achieveing US students: Night owls have higher GMAT scores than early morning types in a top-ranked MBA program. Intelligence. 2014; 47:107-112.

40. Roberts RD, Kyllonen PC. Morningness-eveningness and intelligence: early to bed, early to rise will likely make you anything but wise! Pers Individ Differ. 1999; 27:1123-33.

41. Gale C, Martyn C. Larks and owls and health, wealth, and wisdom. BMJ. 1998; 317:1675-7. [PubMed: 9857121]

42. Killgore WD, Killgore DB. Morningness-eveningness correlates with verbal ability in women but not men. Percept Mot Skills. 2007; 104:335-8. [PubMed: 17450994]

43. Rosenberg J, Maximov II, Reske M, Grinberg F, Shah NJ. "Early to bed, early to rise": diffusion tensor imaging identifies chronotype-specificity. Neuroimage. 2014; 84:428-34. [PubMed: 24001455]

44. Lane JM, Vlasac I, Anderson SG, et al. Genome-wide association analysis identifies novel loci for chronotype in 100,420 individuals from the UK Biobank. Nat Commun. 2016; 7:10889. [PubMed: 26955885]

45. Preckel F, Lipnevich AA, Schneider S, Roberts RD. Chronotype, cognitive abilities, and academic achievement: A meta-analytic investigation. Learn Individ Differ. 2011; 21:483-92.

46. Ferrie JE, Shipley MJ, Akbaraly TN, Marmot MG, Kivimäki M, Singh-Manoux A. Change in sleep duration and cognitive function: findings from the Whitehall II Study. Sleep. 2011; 34:56573. [PubMed: 21532949]

47. Vermeeren A. Residual effects of hypnotics: epidemiology and clinical implications. CNS Drugs. 2004; 18:297-328. [PubMed: 15089115]

48. Curran HV, Collins R, Fletcher S, Kee SC, Woods B, Iliffe S. Older adults and withdrawal from benzodiazepine hypnotics in general practice: effects on cognitive function, sleep, mood and quality of life. Psychol Med. 2003; 33:1223-37. [PubMed: 14580077]

49. Davies G, Marioni RE, Liewald DC, Hill WD, et al. Genome-wide association study of cognitive function and educational attainment in UK biobank $(\mathrm{N}=112,151)$. Molecular Psychiatry. 2015; 21:758-67.

50. Luik AI, Zuurbier LA, Hofman A, Van Someren EJ, Ikram MA, Tiemeier H. Associations of the 24-h activity rhythm and sleep with cognition: a population-based study of middle-aged and elderly persons. Sleep Med. 2015; 16:850-5. [PubMed: 26028055]

51. Swanson JM. The UK biobank and selection bias. The Lancet. 2012; 380:14-20. 


\section{Highlights}

- $\quad$ how different aspects of sleep health relate to cognition is unclear

- we examined sleep and cognitive performance in $\sim 500,000$ adults aged $40-69$ years

- $\quad$ unexpectedly, frequent insomnia symptoms were independently associated with better cognitive performance (effect size differences were very small)

- $\quad$ sleep medication, and long/short sleep duration were linked to cognitive impairment

- morningness was linked to impairment, while eveningness showed superior performance 


\section{Table 1}

Sociodemographic and clinical data for study sample. Groups differed significantly for all variables $(\mathrm{p}<.001)$.

\begin{tabular}{lcc} 
& $\begin{array}{c}\text { Subgroup with frequent insomnia } \\
\text { symptoms }\end{array}$ & $\begin{array}{c}\text { Subgroup without frequent Insomnia } \\
\text { symptoms }\end{array}$ \\
\hline $\mathrm{N}$ & 133,314 & 344,215 \\
Age (yrs) & $57.4 \pm 7.7$ & $56.1 \pm 8.2$ \\
Sex (\% female) & $62.1 \%$ & $52.0 \%$ \\
Sleep duration (hrs) & $6.7 \pm 1.3$ & $7.3 \pm 1.0$ \\
Sleep medication (\%) & $2.1 \%$ & $0.4 \%$ \\
Education (\% with university/ college degree) & $35.9 \%$ & $41.1 \%$ \\
Socioeconomic status $*$ & $-1.16 \pm 3.16$ & $-1.39 \pm 3.04$ \\
BMI (kg/m ${ }^{2}$ ) & $27.8 \pm 5.1$ & $27.2 \pm 4.6$ \\
Hypertension (\%) & $29.9 \%$ & $23.8 \%$ \\
Antihypertensive medication (\%) & $25.4 \%$ & $19.8 \%$ \\
Cardiovascular disease (\%) & $11.0 \%$ & $7.9 \%$ \\
Depressive symptoms $(\%)$ & $34.5 \%$ & $19.1 \%$ \\
Psychotropic medication $(\%)$ & $11.0 \%$ & $5.8 \%$ \\
\hline
\end{tabular}

BMI: body mass index;

Townsend Index of Material Deprivation. 


\section{Table 2}

Unadjusted and multivariate-adjusted relationships between insomnia status and cognitive performance. $\beta$ values indicate unadjusted (model 1) and adjusted (models 2, 3 and 4) group differences between those with frequent insomnia symptoms and those without frequent insomnia symptoms.

\begin{tabular}{|l|l|l|l|l|}
\hline Cognitive Domain & Model 1\# & Model 2\#\# & Model 3\#\#\# & Model 4\#\#\# \\
\hline Reasoning & $\beta=-1.2 \times 10^{-1} * * *$ & $\beta=1.9 \times 10^{-2}$ & $\beta=5.9 \times 10^{-2} * * *$ & $\beta=9.5 \times 10^{-2} * * *$ \\
\hline & $(\mathrm{n}=158,180)$ & $(\mathrm{n}=134,295)$ & $(\mathrm{n}=115,935)$ & $(\mathrm{n}=115,668)$ \\
\hline Reaction Time & $\beta=1.4 \times 10^{-2} * * *$ & $\beta=-2.2 \times 10^{-3} * *$ & $\beta=-5.9 \times 10^{-3} * * *$ & $\beta=-6.7 \times 10^{-3} * * *$ \\
\hline & $(\mathrm{n}=473,144)$ & $(\mathrm{n}=386,588)$ & $(\mathrm{n}=333,095)$ & $(\mathrm{n}=332,303)$ \\
\hline Numeric memory & $\beta=-1.0 \times 10^{-1} * * *$ & $\beta=-1.8 \times 10^{-2}$ & $\beta=4.9 \times 10^{-3}$ & $\beta=1.6 \times 10^{-2}$ \\
\hline & $(\mathrm{n}=48,091)$ & $(\mathrm{n}=40,151)$ & $(\mathrm{n}=34,844)$ & $(\mathrm{n}=34,753)$ \\
\hline Visual Memory & $\beta=1.4 \times 10^{-2} * * *$ & $\beta=-9.0 \times 10^{-3} * *$ & $\beta=-1.3 \times 10^{-2} * * *$ & $\beta=-1.4 \times 10^{-2} * * *$ \\
\hline & $(\mathrm{n}=473,955)$ & $(\mathrm{n}=372,318)$ & $(\mathrm{n}=334,779)$ & $(\mathrm{n}=333,966)$ \\
\hline Prospective Memory & $\beta=-2.7 \times 10^{-2}$ & $\beta=6.1 \times 10^{-2} * * *$ & $\beta=1.1 \times 10^{-1} * * *$ & $\beta=1.5 \times 10^{-1} * * *$ \\
\hline & $(\mathrm{n}=163,077)$ & $(\mathrm{n}=136,770)$ & $(\mathrm{n}=117,721)$ & $(\mathrm{n}=117,438)$ \\
\hline
\end{tabular}

\# unadjusted analyses;

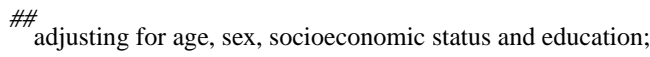

\#\#\# adjusting for age, sex, socioeconomic status, education, BMI, hypertension, cardiovascular disease, antihypertensive medication, depressive symptoms, psychotropic medication, sleep medication, chronotype;

$\# \# \#$ adjusting for Model 3 variables plus sleep duration.

p $<0.01$;

** $\mathrm{p}<0.001$;

$* * *$

$\mathrm{p}<0.0001$ 


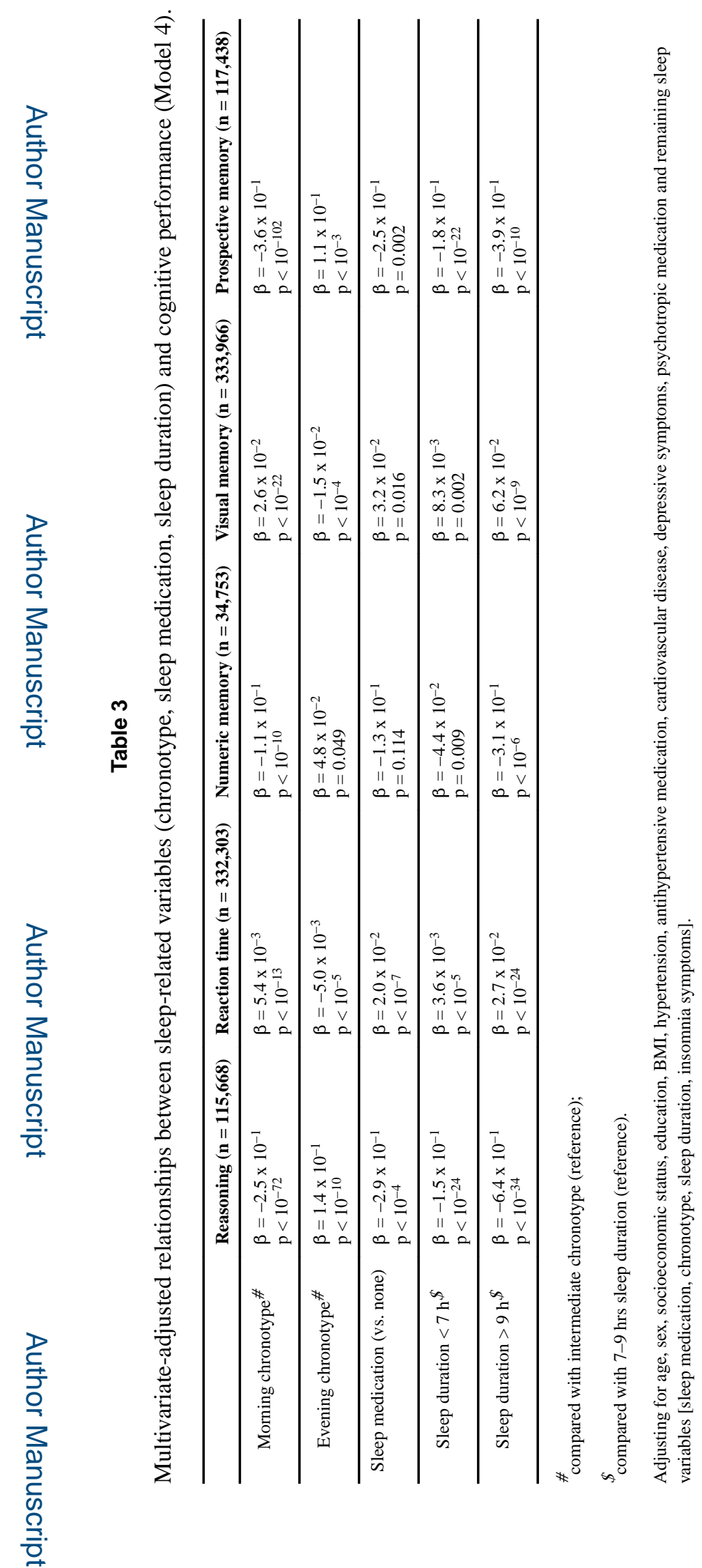

Sleep Med. Author manuscript; available in PMC 2018 May 02. 